1 Hacettepe Journal of Mathematics and Statistics

$\bigcap$ Volume 47 (1) (2018), $1-7$

\title{
A recurrent set for one-dimensional dynamical systems
}

\author{
Seyyed Alireza Ahmadi*
}

\begin{abstract}
In this note we introduce a new kind of recurrent set for a dynamical system on the interval $[0,1]$. This set is not necessarily invariant under continuous conjugacies, but it is invariant under absolutely continuous ones.
\end{abstract}

Keywords: Recurrent set, Chain recurrent set, Absolutely continuous map, Inverse limit space.

2000 AMS Classification: 54H20, 37B40

Received: 22.11.2016 Accepted: 23.02.2017 Doi : 10.15672/HJMS.2017.454

\section{Introduction}

One of the main problems in dynamical systems is the description of the orbit structure of a system from a topological point of view $[6,7,9]$. Recurrence behavior is one of the most important concepts in topological dynamics. An equilibrium solution or periodic solution of a differential equation exhibits recurrence in the sense that it returns to it's initial condition infinitely often. However, solution of differential equations may also tend toward solution displaying more complicated recurrent behaviour. To describe these types of asymptotic behaviours, we seek to understand more complicated forms of recurrence [4]. We shall investigate these behaviors in one-dimensional discrete dynamical systems. By a dynamical system $(I, f)$ we mean that $I=[0,1]$ and $f: I \rightarrow I$ is a continuous map. The following examples of recurrent sets are well-known [2].

- A fixed point of dynamical system $(I, f)$, exhibits the simplest type of recurrence. We denote by $\operatorname{Fix}(f)$ the set of all fixed points of $f$.

- A point carried back to itself by a dynamical system $(I, f)$ exhibits the next most elementary type of recurrence. A point $x \in I$ is called periodic if there exists $n \in \mathbb{N}$ such that $f^{n}(x)=x$. We denote by $\operatorname{Per}(f)$ the set of all periodic points of $f$.

${ }^{*}$ Department of Mathematics, University of Sistan and Baluchestan, Zahedan, Iran., Email: sa.ahmadi@math.usb.ac.ir, sa.ahmdi@gmail.com 
- A major object in the study of dynamical systems is to describe the eventual behavior of an orbit $\left\{f^{n}(x)\right\}_{n \in \mathbb{Z}}$. A point $x \in I$ is called an $\omega$-limit point for $x \in I$ provided that there exists a sequence $n_{k} \rightarrow \infty$, (as $\left.k \rightarrow \infty\right)$ such that $f^{n_{k}}(x) \rightarrow y$. A point $y \in I$ is called an $\alpha$-limit point of $x \in I$ provided that there exists a sequence $n_{k} \rightarrow \infty$, (as $\left.k \rightarrow \infty\right)$ such that $f^{-n_{k}}(x) \rightarrow y$. We denote by $\omega(x)$ and $\alpha(x)$ the set of all $\omega$-limit points and $\alpha$-limit points, respectively. A point $x \in I$ is called Poincare recurrent if $x \in \omega(x) \cap \alpha(x)$. We denote by $\mathcal{R}(f)$ the set of all Poincare recurrent points of $f$.

- A point $x \in I$ is non-wandering if for each neighborhood $U$ of $x$, there exists $n \in \mathbb{N}$ such that $U \cap f^{n}(U) \neq \emptyset$. We denote by $\Omega(f)$ the set of all non-wandering points of $f$.

- An $\epsilon$-pseudo-orbit (or $\epsilon$-chain) of $f$ from $x$ to $y$ is a sequence $\left\{x_{i}\right\}_{i=0}^{n}$ with $x_{0}=x$, $x_{n}=y$ and $\left|f\left(x_{k}\right)-x_{k+1}\right|<\epsilon$, for all $k=0,1, \ldots, n-1$. A point $x$ in $I$ is called chain recurrent if for each $\epsilon>0$, there is an $\epsilon$-chain from $x$ to itself. We denote by $\mathcal{C R}(f)$ the set of all chain recurrent points of $f$.

It is known that

$$
\operatorname{Fix}(f) \subseteq \operatorname{Per}(f) \subseteq \mathcal{R}(f) \subseteq \Omega(f) \subseteq \mathcal{e} \mathcal{R}(f) .
$$

By denoting $\mathfrak{R}(f)$, as a recurrent set, almost all of the aforementioned recurrent sets have the following desirable properties:

(1) The set $\mathfrak{R}(f)$ is forward invariant with respect to $f$, that is $f(\mathfrak{R}(f)) \subseteq \mathfrak{R}(f)$.

(2) $\mathfrak{R}(f)$ is closed.

(3) $\mathfrak{R}(f)$ is invariant under topological conjugacy, that is, if $(I, f)$ and $(J, g)$ are two dynamical systems and $h: I \rightarrow J$ is a homeomorphism with $h \circ f=g \circ h$, then $\mathfrak{R}(g)=h(\Re(f))$.

(4) $\mathfrak{R}(f)$ has the restriction property, that is $\mathfrak{R}\left(\left.f\right|_{\mathfrak{R}(f)}\right)=\mathfrak{R}(f)$

Ideally, a recurrent set must have the properties (1)-(4). In this paper we are going to introduce and study a new type of recurrence for an absolutely continuous dynamical system.

\section{Preliminaries}

We use the symbol $\mathcal{O}_{\delta}(f, x, y)$ for the set of $\delta$-pseudo-orbits $\left\{x_{i}\right\}_{i=0}^{n}$ of $f$ with $x_{0}=x$ and $x_{n}=y$. For given points $x, y \in X$ we write $x \stackrel{\epsilon}{\rightsquigarrow} y$ if $\mathcal{O}_{\epsilon}(f, x, y) \neq \emptyset$ and we write $x \rightsquigarrow y$ if $\mathcal{O}_{\epsilon}(f, x, y) \neq \emptyset$ for each $\epsilon>0$. We write $x \rightsquigarrow y$ if $x \rightsquigarrow y$ and $y \rightsquigarrow x$. The set $\{x \in I: x \leftrightarrow x\}$ is called the chain recurrent set of $f$ and is denoted by $\mathcal{C R}(f)$. If we define a relation $R$ on $I$ with $x R y \Leftrightarrow x \leftrightarrow y$, then $R$ is an equivalence relation on eR $(f)$.

A dynamical system $(I, f)$ is called chain recurrent if $\mathcal{C} \mathcal{R}(f)=I$. A dynamical system $f$ is called chain transitive if for every $x, y \in I$ we deduce $x \leftrightarrow y$.

We say that a dynamical system $(I, f)$ has the shadowing property on $X$ if for each $\epsilon>0$ there is $\delta>0$ such that for a given sequence $\xi=\left\{x_{k}\right\}_{n \in \mathbb{Z}}$ with

$$
\left|f\left(x_{k}\right)-x_{k+1}\right|<\delta \quad \text { for } k \in \mathbb{N},
$$

there exists a point $x \in I$ such that

$$
\left|f^{k}(x)-x_{k}\right|<\epsilon \quad \text { for } k \in \mathbb{N}
$$

(In this case we say that $x \in I, \epsilon$-shadows $\xi$ ).

Recall that a function $f: I \rightarrow \mathbb{R}$ is called absolutely continuous if for every $\varepsilon>$ 0 there is $\delta>0$ such that, for every finite collection of pairwise disjoint intervals 
$\left(a_{1}, b_{1}\right),\left(a_{2}, b_{2}\right), \ldots,\left(a_{n}, b_{n}\right) \subset I$ with $\sum_{i}\left|a_{i}-b_{i}\right|<\delta$, we have

$$
\sum_{i}\left|f\left(b_{i}\right)-f\left(a_{i}\right)\right|<\varepsilon
$$

For most of the existing instances of absoloutely continuous functions, the inverse function is also absoloutely continuous. The paper [8] presents a function which shows that this is not true in general.

In following definition from [1] allows us to define our main concept in the next section.

2.1. Definition. Let $(I, f)$ be a dynamical system. Given $\epsilon>0$ we define a function $\delta_{f, \epsilon}: I \times I \rightarrow[0, \infty)$ by

$$
\delta_{f, \epsilon}(x, y)=\inf \left\{\sum_{i=0}^{k}\left|p_{i}-q_{i}\right|: p_{0}=x, q_{k}=y, k \in \mathbb{N}\right\}, x, y \in I
$$

where the infimum is taken over all choices of $p_{i}$ and $q_{i}$ so that $q_{i} \stackrel{\epsilon}{\leftrightarrow} p_{i+1}$ for all $i=0,1, \ldots, k-1$. Obviously the points $p_{i}$ and $q_{i}$ can be chosen such that the intervals $\left(p_{i}, q_{i}\right)$ or $\left(q_{i}, p_{i}\right)$ be pairwise disjoint.

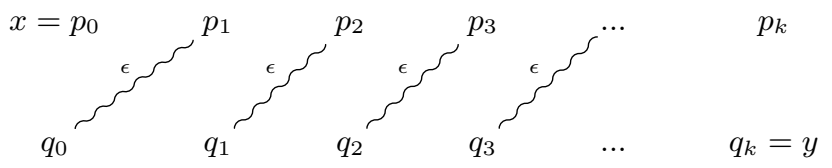

Obviously for any $\epsilon>0$ the function $\delta_{f, \epsilon}$ is a pseudo-metric on $I$. We denote by $\tau_{f, \epsilon}$ the topology induced by this pseudo-metric. If $\mathcal{C R}(f)=I$, then $\tau_{f, \epsilon}$ is finer that standard topology on $I$.

\section{Main results}

In this section we are going to introduce a new kind of recurrent set for dynamical system $(I, f)$.

3.1. Definition. We say that a point $x \in I$ is weak chain recurrent if $\delta_{f, \epsilon}(x, x)<\epsilon$ for all $\epsilon>0$. We denote by $\Delta(f)$ the set of all weak chain recurrent points.

Obviously

$$
\operatorname{Fix}(f) \subseteq \operatorname{Per}(f) \subseteq \mathcal{R}(f) \subseteq \Omega(f) \subseteq \mathcal{C R}(f) \subseteq \Delta(f)
$$

Now we prove the desirable properties of recurrent sets for $\Delta(f)$. We begin with Invariance property.

3.2. Proposition. If $f$ is absolutely continuous then $\Delta(f)$ is forward invariant.

Proof: Given $\epsilon>0$ there exists $\delta>0$ such that for each $n \in \mathbb{N}$ and every finite collection of pairwise disjoint intervals $\left(x_{1}, y_{1}\right), \ldots\left(x_{n}, y_{n}\right)$ in $I$ the inequality $\sum_{i=1}^{n} \mid x_{i}-$ $y_{i} \mid<\delta$ implies $\sum_{i=1}^{n}\left|f\left(x_{i}\right)-f\left(y_{i}\right)\right|<\epsilon$. Suppose that $x \in \Delta(f)$, then $\delta_{f, \delta}(x, x)<\delta$. Thus there exist intervals $\left(p_{0}, q_{0}\right), \ldots\left(p_{k}, q_{k}\right), k \in \mathbb{N}$ such that $\sum_{i=0}^{k}\left|p_{i}-q_{i}\right|<\delta, q_{i} \stackrel{\delta}{\rightsquigarrow} p_{i+1}$ for $i=0,1, \ldots k-1$ and $p_{0}=x, q_{k}=x$. Hence $\sum_{i=0}^{n}\left|f\left(p_{i}\right)-f\left(q_{i}\right)\right|<\epsilon$, moreover $f\left(q_{i}\right) \stackrel{\epsilon}{\leftrightarrow} f\left(p_{i+1}\right)$ for $i=0,1, \ldots k-1$ and $f\left(p_{0}\right)=f(x), f\left(q_{k}\right)=f(x)$. Therefore $\delta_{f, \epsilon}(f(x), f(x))<\epsilon$. That is $f(x) \in \Delta(f)$.

3.3. Proposition. $\Delta(f)$ is closed. 
Proof: Suppose that $x \in \overline{\Delta(f)}$ we shall show that $x \in \Delta(f)$. Given $\epsilon>0$ there exists a point $y \in \Delta(f)$ such that $|x-y|<\epsilon / 3$. Since $y \in \Delta(f)$ we obtain $\delta_{f, \epsilon / 3}(y, y)<\epsilon / 3$. Thus there exist intervals $\left(p_{0}, q_{0}\right) \ldots\left(p_{k}, q_{k}\right), k \in \mathbb{N}$, such that $\sum_{i=0}^{k}\left|p_{i}-q_{i}\right|<\epsilon / 3, q_{i} \stackrel{\epsilon / 3}{\leftrightarrow m} p_{i+1}$ for $i=0,1, \ldots k-1$ and $p_{0}=y, q_{k}=y$. Define

$$
p_{i}^{\prime}=\left\{\begin{array}{cll}
x & \text { if } \quad i=0 \\
p_{i} & \text { if } \quad i=1,2, \ldots k
\end{array}\right.
$$

and

Therefore

$$
q_{i}^{\prime}=\left\{\begin{array}{cll}
q_{i} & \text { if } & i=0,1, \ldots k-1 \\
x & \text { if } & i=k
\end{array}\right.
$$

$$
\sum_{i=0}^{k}\left|p_{i}^{\prime}-q_{i}^{\prime}\right| \leq 2|x-y|+\sum_{i=0}^{k}\left|p_{i}-q_{i}\right|<\epsilon .
$$

Moreover $p_{0}^{\prime}=x, q_{k}^{\prime}=x$ and $q_{i}^{\prime} \stackrel{\epsilon}{\rightsquigarrow} p_{i+1}^{\prime}$ for $i=0,1, \ldots k-1$. Hence $\delta_{f, \epsilon}(x, x)<\epsilon$. That is $x \in \Delta(f)$.

3.4. Theorem. Let $(I, f)$ and $(J, g)$ be two dynamical systems and suppose that $h: I \rightarrow J$ is an absolutely continuous map with absolutely continuous inverse such that $h \circ f=g \circ h$. Then $h(\Delta(f))=\Delta(g)$.

Proof. Assume that $z=h(x) \in h(\Delta(f))$ and $\xi>0$. Suppose that $\eta$ is a $\xi$ modulus of absolute continuity of $h$. Since $x \in \Delta(f)$, we obtain $\delta_{f, \eta}(x, x)<\eta$. Thus there exist a finite collection of pairwise disjoint intervals $\left(p_{0}, q_{0}\right), \ldots,\left(p_{k}, q_{k}\right)$ such that $\sum_{i=0}^{k} \mid p_{i}-$ $q_{i} \mid<\eta$ and $q_{i} \stackrel{\eta}{\leftrightarrow} p_{i+1}$ for all $i=0,1, \ldots, k-1$. Hence $\sum_{i=0}^{k}\left|h\left(p_{i}\right)-h\left(q_{i}\right)\right|<\xi$ and $h\left(q_{i}\right) \stackrel{\xi}{m} h\left(p_{i+1}\right)$. Therefore $\delta_{g, \xi}(h(x), h(y))<\xi$ and $z \in \Delta(g)$. A similar proof works for the inclusion $h(\Delta(f)) \supseteq \Delta(g)$.

3.5. Lemma. Let $(I, f)$ be a dynamical system. Then for any $\epsilon>0$ there exists $\delta>0$ such that for each $x, y \in I$,

$$
\mathcal{O}_{\delta}(f, x, y) \neq \emptyset \Rightarrow \mathcal{O}_{\epsilon}\left(\left.f\right|_{\mathcal{e} R(f)}, x, y\right) \neq \emptyset .
$$

Proof. By contrapositive assume that there exists $\epsilon>0$ such that for any $n \in \mathbb{N}$ there exist points $x_{n}, y_{n} \in I$ with $\mathcal{O}_{\frac{1}{n}}(f, x, y) \neq \emptyset$ such that $\mathcal{O}_{\epsilon}\left(f, x_{n}, y_{n}\right)=\emptyset$. Passing to the subsequnces we can assume that $x_{n} \rightarrow x$ and $y_{n} \rightarrow y$ for some $x, y \in I$. For each $n \in \mathbb{N}$, there exists a sequence $x_{n}=x_{n, 0}, x_{n, 1}, \ldots x_{n, k_{n}}=y_{n}$ such that $\left|f\left(x_{n, i}\right)-x_{n, i+1}\right|<\frac{1}{n}$ for all $i=0,1, \ldots, k_{n}-1$. Define $C_{n}=\left\{x_{n, 0}, x_{n, 1}, \ldots, x_{n, k_{n}}\right\}$ for each $n \in \mathbb{N}$, so the set $C_{n}$ is compact . The space $K(I)$ of all nonempty compact subsets of $I$ with the Hausdorff metric

$$
D(A, B)=\sup \{|a-B|,|b-A|: a \in A, b \in B\}
$$

is a compact metric space. Thus the sequence $\left\{C_{n}\right\}$ in $K(I)$ contains a sub-sequence $C_{n_{l}}$ which converges to some $C \in K(I)$. We may assume, without loss of generality, that $\left\{C_{n}\right\}$ converges to $C$.

Assume that $p, q \in C$ and $\delta \in\left(0, \frac{\epsilon}{3}\right)$ is an $\epsilon$ modulus of uniform continuity of $f$. Since $\left\{C_{n}\right\}$ converges to $C$ there exists a positive integer $n$ such that $\frac{1}{n}<\epsilon$ and $D\left(C_{n}, C\right)<\delta$. Hence there exist $x_{n, s}, x_{n, t} \in C_{n}$ such that $\left|x_{n, s}-p\right|<\delta,\left|x_{n, t}-q\right|<\delta$ and $x_{n+s}<x_{n_{t}}$. By defining

$$
y_{i}=\left\{\begin{array}{cl}
x_{n, s+i} & \text { if } \quad i=0,1, \ldots, k_{n}-s-1, \\
x_{n, s+i-k_{n}} & \text { if } i=k_{n}-s \ldots k_{n} .
\end{array}\right.
$$

Since $D\left(C_{n}, C\right)<\delta$, for each $j=0,1, \ldots, k_{n}-1$ there exists $p_{j} \in C$ such that $\left|y_{j}-p_{j}\right|<\delta$. Put $p_{0}=p$ and $p_{k_{n}}=q$, obviously $p=p_{0}, p_{1}, \ldots, p_{k_{n}}=q$ is an $\epsilon$-pseudo-orbit from $p$ to 
$q$. Consequently $p_{0}, p_{1}, \ldots p_{k_{n}}$ is an $\epsilon$-pseudo-orbit in $C$ from $p$ to $q$. Since $x_{n}, y_{n} \in C_{n}$ for each $n \in \mathbb{N}$, we obtain that $x, y \in C$. Therefore $\mathcal{O}_{\epsilon}\left(\left.f\right|_{\mathcal{C R}(f)}, x_{n}, y_{n}\right) \neq \emptyset$ for some $n \in \mathbb{N}$ which is a contradiction.

3.6. Theorem. Let $(I, f)$ be a dynamical system. then $\Delta\left(\left.f\right|_{\Delta(f)}\right)=\Delta(f)$.

Proof. The inclusion $\Delta\left(\left.f\right|_{\Delta(f)}\right) \subseteq \Delta(f)$ is immediate. Assume that $x \in \Delta(f)$. Given $\xi>0$, by Lemma 3.5 there exists $\eta \in(0, \xi)$ such that for all $x, y \in I$,

$$
\mathcal{O}_{\eta}(f, x, y) \neq \emptyset \Rightarrow \mathcal{O}_{\xi}\left(\left.f\right|_{\mathcal{C R}(f)}, x, y\right) \neq \emptyset .
$$

Since $\delta_{f, \eta}(x, x)<\eta$, there exist points $p_{0}, p_{1}, \ldots, p_{k}, q_{0}, q_{1}, \ldots, q_{k}$ with $p_{0}=x, q_{k}=x$ such that $q_{i} \stackrel{\eta}{m} p_{i+1}$ with respect to $f$ and $\sum_{i=0}^{k}\left|p_{i}-q_{i}\right|<\eta$. Therefore $\mathcal{O}_{\eta}\left(f, q_{i}, p_{i+1}\right) \neq$ $\emptyset$ and by 3.1 we obtain $\mathcal{O}_{\xi}\left(\left.f\right|_{\mathcal{E R}(f)}, q_{i}, p_{i+1}\right) \neq \emptyset$. This implies that $x \in \Delta\left(\left.f\right|_{\Delta(f)}\right)$.

\section{Weak chain recurrent set in metric spaces}

If $(X, d)$ is a compact metric space and $f: X \rightarrow X$ is continuous, then for every $x, y \in X$, we can define

$$
\delta_{f, \epsilon}(x, y)=\inf \left\{\sum_{i=0}^{k} d\left(p_{i}, q_{i}\right): p_{0}=x, q_{k}=y, k \in \mathbb{N}\right\}
$$

where the infimum is taken over all choices of $p_{i}$ and $q_{i}$ so that $q_{i} \stackrel{\epsilon}{\leftrightarrow} p_{i+1}$ for all $i=0,1, \ldots, k-1$.

We also define

$$
\delta_{f}(x, y)=\inf \left\{\sum_{i=0}^{k} d\left(p_{i}, q_{i}\right): p_{0}=x, q_{k}=y, k \in \mathbb{N}\right\}
$$

where the infimum is taken over all choices of $p_{i}$ and $q_{i}$ so that $q_{i}$ « $p_{i+1}$ for all $i=0,1, \ldots, k-1$.

The straightforward calculations imply that for $\epsilon_{1} \leq \epsilon_{2}$ we deduce $\delta_{f, \epsilon_{2}}(x, y) \leq \delta_{f, \epsilon_{1}}(x, y) \leq$ $\delta_{f}(x, y)$.

In [1] the author proved the following theorems.

4.1. Theorem. [1] Let $f: X \rightarrow X$ be a chain recurrent continuous map. Then $f$ is chain transitive if and only if $\delta_{f}(x, y)=0$ for all $x, y \in X$.

4.2. Theorem. [1] Let $f: X \rightarrow X$ be a chain recurrent continuous map. Then $\delta_{f}(x, y)=$ $d(x, y)$ for all $x, y \in X$ if and only if $f$ is the identity map and $X$ is totally disconnected.

4.3. Theorem. [1] Let $(X, d)$ be a compact metric space. Then $X$ is connected if and only if for each $x, y \in X, \delta_{i d}(x, y)=0$.

Let $f: X \rightarrow X$ be a continuous surjection. Then if

$$
\lim _{\leftarrow}(I, f)=\left\{\boldsymbol{x}=\left(x_{i}\right): x_{i} \in X \text { and } f\left(x_{i+1}\right)=x_{i}, i \geq 0\right\}
$$

and

$$
\rho\left(\left(x_{i}\right),\left(y_{i}\right)\right)=\sum_{i=0}^{\infty} \frac{\left|x_{i}-y_{i}\right|}{2^{i}}
$$

then $\left(X_{f}, \rho\right)$ is a metric space called inverse limit space. The homeomorphism $\sigma: X_{f} \rightarrow$ $X_{f}$ with $\sigma\left(\left(x_{i}\right)_{i=0}^{\infty}\right)=\left(f\left(x_{i}\right)\right)_{i=0}^{\infty}$ is called the shift map. Investigating the relationships between the dynamical properties of continuous map and shift map of the inverse limit space is always interesting [5]. 
4.4. Theorem. [3] Let $f: I \rightarrow I$ be a continuous surjection and let $\lim _{\leftarrow}(I, f)$ be the inverse limit space with the shift map $\sigma$. Then

(1) $F i x(\sigma)=\lim _{\leftarrow}(F i x(f), f)$

(2) $\operatorname{Per}(\sigma)=\lim _{\leftarrow}(\operatorname{Per}(f), f)$

(3) $\mathcal{R}(\sigma)=\lim _{\leftarrow}(\mathcal{R}(f), f)$

(4) $\Omega(\sigma)=\lim _{\leftarrow}(\Omega(f), f)$

(5) $\mathcal{C R}(\sigma)=\lim _{\leftarrow}(\mathcal{C R}(f), f)$

Now we are going to prove the same statement for $\Delta(f)$.

4.5. Theorem. Let $f: I \rightarrow I$ be a continuous surjection and $\Delta(\sigma)$ be the weak chain recurrent set for the shift map $\sigma: \lim _{\leftarrow}(I, f) \rightarrow \lim _{\leftarrow}(I, f)$. Then we deduce $\Delta(\sigma)=$ $\left(\lim _{\leftarrow}(\Delta(f), f)\right.$

Proof. First we prove the inclusion $\Delta(\sigma) \subseteq\left(\lim _{\leftarrow}(\Delta(f), f)\right.$. Suppose that $\left(x_{i}\right) \in \Delta(\sigma)$. Fix $m \geq 0$, for $\epsilon>0$, since $\delta_{\sigma, 2^{-m} \epsilon}\left(\left(x_{i}\right),\left(x_{i}\right)\right)<2^{-m} \epsilon$ there exist points $\left(p_{i}^{j}\right),\left(q_{i}^{j}\right) \epsilon$ $\lim _{\leftarrow}(I, f), j=0,1, \ldots, k$ such that $\left(p_{i}^{0}\right)=\left(x_{i}\right),\left(q_{i}^{k}\right)=\left(x_{i}\right),\left(q_{i}^{j}\right)^{2_{\leftarrow m}^{-m} \epsilon}\left(p_{i}^{j+1}\right)$ for $j=$ $0,1, \ldots, k-1$ and

$$
\sum_{j=0}^{k} \rho\left(\left(p_{i}^{j}\right),\left(q_{i}^{j}\right)\right)<\frac{\epsilon}{2^{m}} .
$$

We show that for each $j=0,1, \ldots k-1, q_{m}^{j} \stackrel{\epsilon}{\leftrightarrow} p_{m}^{j+1}$. Fix $j \geq 0$, then $\mathcal{O}_{\epsilon / 2^{m}}\left(\sigma,\left(q_{i}^{j}\right),\left(p_{i}^{j+1}\right)\right) \neq$ $\emptyset$. Let $\left\{\left(r_{i}^{0}\right),\left(r_{i}^{1}\right), \ldots,\left(r_{i}^{n}\right)\right\} \in \mathcal{O}_{\epsilon / 2^{m}}\left(\sigma,\left(q_{i}^{j}\right),\left(p_{i}^{j+1}\right)\right)$. Then

$$
\frac{\left|f\left(r_{m}^{l}\right)-r_{m}^{l+1}\right|}{2^{m}}=\frac{\left|r_{m-1}^{l}-r_{m}^{l+1}\right|}{2^{m}} \leq \rho\left(\sigma\left(r_{i}^{l}\right),\left(r_{i}^{l+1}\right)\right)<\epsilon / 2^{m}
$$

for $l=0,1, \ldots, n-1$. Thus $\left\{r_{m}^{0}, r_{m}^{1}, \ldots, r_{m}^{n}\right\} \in \mathcal{O}_{\epsilon}\left(f, q_{m}^{j}, p_{m}^{j+1}\right)$ so $q_{m}^{j} \stackrel{\epsilon}{\leftrightarrow} p_{m}^{j+1}$. On other hand

$$
\sum_{j=0}^{k} \sum_{i=0}^{\infty} \frac{\left|p_{i}^{j}-q_{i}^{j}\right|}{2^{i}}<\frac{\epsilon}{2^{m}}
$$

Hence

$$
\sum_{j=0}^{k}\left|p_{m}^{j}-q_{m}^{j}\right|<\epsilon
$$

Therefore $\delta_{f, \epsilon}\left(x_{m}, x_{m}\right)<\epsilon$. This implies that $x_{m} \in \Delta(f)$. Since $m$ is arbitrary we obtain $\left(x_{i}\right) \in\left(\lim _{\leftarrow}(\Delta(f), f)\right.$.

Conversely assume that $\boldsymbol{x}=\left(x_{i}\right) \in \lim _{\leftarrow}(\Delta(f), f)$. Given $\epsilon>0$, there exists $m>0$ such that $2^{-m+1}<\epsilon$, by uniform continuity of $f$ there exists $\eta \in(0, \epsilon / 4)$ such that $|x-y|<\eta$ implies that

$$
\max _{0<i \leq m}\left|f^{i}(x)-f^{i}(y)\right|<\epsilon / 4 .
$$

Since $x_{m} \in \Delta(f)$, we obtain $\delta_{f, \eta}\left(x_{m}, x_{m}\right)<\eta$. Then there exist points $x_{m}=p_{m}^{0}, p_{m}^{1}, \ldots, p_{m}^{k}$ and $q_{m}^{0}, q_{m}^{1}, \ldots, q_{m}^{k}=x_{m}$ in $\Delta(f)$ such that $q_{m}^{j} \stackrel{\delta}{\leftrightarrow} p_{m}^{j+1}$ for all $j=0,1, \ldots, k-1$, and $\sum_{j=0}^{k}\left|p_{m}^{j}-q_{m}^{j}\right|<\delta$.

Fix $j$, since $q_{m}^{j} \stackrel{\delta}{\leftrightarrow} p_{m}^{j+1}$, we obtain $\mathcal{O}_{\delta}\left(f, q_{m}^{j}, p_{m}^{j+1}\right) \neq \emptyset$. Suppose that $\left\{r_{m}^{0}, \ldots r_{m}^{n}\right\} \in$ $\mathcal{O}_{\delta}\left(f, q_{m}^{j}, p_{m}^{j+1}\right) \cap \mathcal{C R}(f)$. Then $\left|f^{i+1}\left(r_{m}^{l}\right)-f\left(r_{m}^{l+1}\right)\right|<\epsilon / 4$ for all $l=0,1, \ldots, n-1$ and all $0<i \leq m$. Hence we conclude that

$$
\sum_{i=0}^{m} \frac{\left|f^{i+1}\left(r_{m}^{l}\right)-f^{i}\left(r_{m}^{l+1}\right)\right|}{2^{m-i}} \leq \sum_{i=0}^{m} \frac{\epsilon}{4} \cdot \frac{1}{2^{m-i}}<\frac{\epsilon}{2} .
$$


Since $f(\mathcal{C R}(f))=\mathcal{C R}(f)$, for $0 \leq l \leq n-1$ we can find points $r_{m+1}^{l}, r_{m+2}^{l}, \ldots$ such that

$$
\boldsymbol{q}^{l}=\left(f^{m}\left(r_{m}^{l}\right), f^{m-1}\left(r_{m}^{l}\right), \ldots, f\left(r_{m}^{l}\right), r_{m}^{l}, r_{m+1}^{l}, r_{m+2}^{l}, \ldots\right) \in \underset{\leftarrow}{\lim }(\mathrm{e} \mathcal{R}(f), f),
$$

$\boldsymbol{q}^{0}=\left(q_{i}^{j}\right)$ and $\boldsymbol{q}^{n}=\left(p_{i}^{j+1}\right)$. Furthermore we obtain

$$
\rho\left(\sigma\left(\boldsymbol{q}^{l}\right), \boldsymbol{q}^{l+1}\right)=\sum_{i=0}^{m} \frac{\left|f^{i+1}\left(r_{m}^{l}\right)-f^{i}\left(r_{m}^{l+1}\right)\right|}{2^{m-i}}+\sum_{i=m+1}^{\infty} \frac{\left|r_{i-1}^{l}-r_{i}^{l+1}\right|}{2^{i}}<\frac{\epsilon}{2}+\frac{1}{2^{m}}<\epsilon .
$$

Hence for fixed $j$, the sequence $\left\{\boldsymbol{q}^{0}, \boldsymbol{q}^{1}, \ldots, \boldsymbol{q}^{n}\right\}$ is an $\epsilon$-chain from $\left(q_{i}^{j}\right)$ to $\left(p_{i}^{j+1}\right)$. That is $\left(q_{i}^{j}\right) \stackrel{\epsilon}{\rightsquigarrow}\left(p_{i}^{j+1}\right)$. Similar proof shows that $\left(p_{i}^{j+1}\right) \stackrel{\epsilon}{\rightsquigarrow}\left(q_{i}^{j}\right)$. Finally we have

$$
\sum_{j=0}^{k} \rho\left(\left(p_{i}^{j}\right),\left(q_{i}^{j}\right)\right)=\sum_{j=0}^{k} \sum_{i=0}^{\infty} \frac{\left|p_{i}^{j}-q_{i}^{j}\right|}{2^{i}} \leq \sum_{i=0}^{\infty} \frac{\epsilon / 2}{2^{i}}=\epsilon .
$$

Therefore $\rho_{\sigma, \epsilon}(\boldsymbol{x}, \boldsymbol{x})<\epsilon$. This completes the proof.

\section{Acknowledgment}

I would like to express my thanks for the invaluable suggestions of Dr. Hossein Hosseini Giv, my friend and my colleague.

\section{References}

[1] S. A. Ahmadi. On the topology of the chain recurrent set of a dynamical system. Applied general topology, 15(2):167-174, 2014.

[2] J. M. Alongi and G. S. Nelson. Recurrence and topology, volume 85. American Mathematical Soc., 2007.

[3] N. Aoki and K. Hiraide. Topological theory of dynamical systems: recent advances, volume 52. Elsevier, 1994.

[4] P. Krupski, K. Omiljanowski, and K. Ungeheuer. Chain recurrent sets of generic mappings on compact spaces. Topology and its Applications, 202:251 - 268, 2016.

[5] S. Li. Dynamical properties of the shift maps on the inverse limit spaces. Ergodic Theory and Dynamical Systems, 12(01):95-108, 1992.

[6] N. Shekutkovski and M. Shoptrajanov. Intrinsic shape of the chain recurrent set. Topology and its Applications, 202:117 - 126, 2016.

[7] T. Shimomura. Special homeomorphisms and approximation for cantor systems. Topology and its Applications, 161:178 - 195, 2014.

[8] S. Spataru. An absolutely continuous function whose inverse functionis not absolutely continuous. Note di Matematica, 23(1):47-49, 2004.

[9] X. Wen and L. Wen. Codimension one structurally stable chain classes. Transactions of the American Mathematical Society, 368(6):3849-3870, 2016. 
